CVIA

CASE REPORT

pISSN 2508-707X / eISSN 2508-7088 https://doi.org/10.22468/cvia.2017.00129 CVIA 2018;2(1):34-36

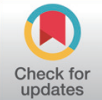

Received: September 1, 2017

Revised: October 9, 2017

Accepted: December 12, 2017

Corresponding author

Johann Christopher, MD, DNB, FACC

Consultant Cardiologist,

Director of Cardiac Imaging,

Care Hospitals, Road no.1, Banjara Hills,

Hyderabad, Telangana 500034, India

Tel: 91-40-30418806

Fax: 91-40-30418488

E-mail: johann1403@gmail.com

\section{Post-Infarct Cardiac Free Wall Rupture Detected by Multi-Detector Computed Tomography}

\author{
Johann Christopher ${ }^{1}$, Chinnam Naidu Sirasapalli² \\ ${ }^{1}$ Consultant Cardiologist, Director of Cardiac Imaging, Care Hospitals, Telangana, India \\ ${ }^{2}$ Department of Radiology, Care Hospitals, Telangana, India
}

\begin{abstract}
A 48-year-old female with chest pain was diagnosed with an inferoposterior wall myocardial infarction. A moderate pericardial effusion was revealed on two-dimensional echocardiography. Post-thrombolysis, the patient experienced persistent chest pain. In view of the possibility of a sealed myocardial rupture, the patient underwent coronary CT angiography (CTA). The rupture was confirmed via CTA, and the coronary anatomy was delineated. The patient underwent pericardial patch repair with good results.
\end{abstract}

Key words Cardiac free wall rupture - Myocardial infarction · CT angiography Pericardial effusion · Pseudoaneurysm.

\section{INTRODUCTION}

A cardiac free wall rupture following myocardial infarction (MI) is a life-threatening complication that often results in the sudden onset of cardiogenic shock caused by cardiac tamponade. Multi-detector computed tomography (MDCT) provides valuable information in a patient suspected to have post-infarct cardiac rupture.

\section{CASE REPORT}

A 48-year-old female with a history of chest pain lasting a duration of four days was admitted to a nearby hospital, where she was diagnosed with inferoposterior wall ST elevation MI and was thrombolyzed with streptokinase. The patient was referred to our hospital for further evaluation due to moderate pericardial effusion .

Examination of the patient revealed a pulse rate of 126 beats/ min, a blood pressure of 100/60 mm Hg, and elevated jugular venous pressure. Cardiovascular examination revealed normal heart sounds with no murmur or rub. Respiratory examination revealed few basal crepitations. Evaluation via ECG showed sinus tachycardia, qS in II and III, and aVF with concomitant ST elevation. There were T inversions together with ST depression

(c) This is an Open Access article distributed under the terms of the Creative Commons Attribution Non-Commercial License (http://creativecommons.org/licenses/bync/4.0) which permits unrestricted non-commercial use, distribution, and reproduction in any medium, provided the original work is properly cited. from V1 to V3.

Two-dimensional (2D) transthoracic echocardiography showed regional wall motion abnormalities in the basal and midinferior walls and the inferior septum and basal posterolateral wall, moderate left ventricular (LV) dysfunction, preserved right ventricular (RV) function, moderate mitral regurgitation, and mild localized pericardial effusion. The patient's blood analysis was unremarkable.

In view of the recent onset pericardial effusion post-thrombolysis and tachycardia with persistent ST elevations, cardiac rupture was suspected. The $2 \mathrm{D}$ echocardiography did not reveal any clue to the cause of pericardial effusion, so an additional investigation was sought to clarify the suspected diagnosis. Coronary CT angiography (CTA) was planned because the process is rapid, noninvasive, and provides information on coronary anatomy. Most importantly, with the cine sequences, the rupture track is delineated, and the contrast pooling in the pericardial space is diagnostic of rupture.

A retrospective gated coronary CT scan was performed, revealing a sealed myocardial rupture in the basal posterolateral wall of the left ventricle with a neck of $2 \mathrm{~mm}$ and an area of 0.8 $\mathrm{cm}^{2}$ (Fig. 1A). Total occlusion of a large obtuse marginal 2 branch with grade $\mathrm{V}$ thrombus was shown on CT coronary angiogram (Fig. 1B). Decreased perfusion was noted on rest perfusion multiplanar images in the vascular territory of the myocardial rupture (Fig. 1C and D). Coronary anatomy was clearly depicted despite the patient's heart rate being above 100 

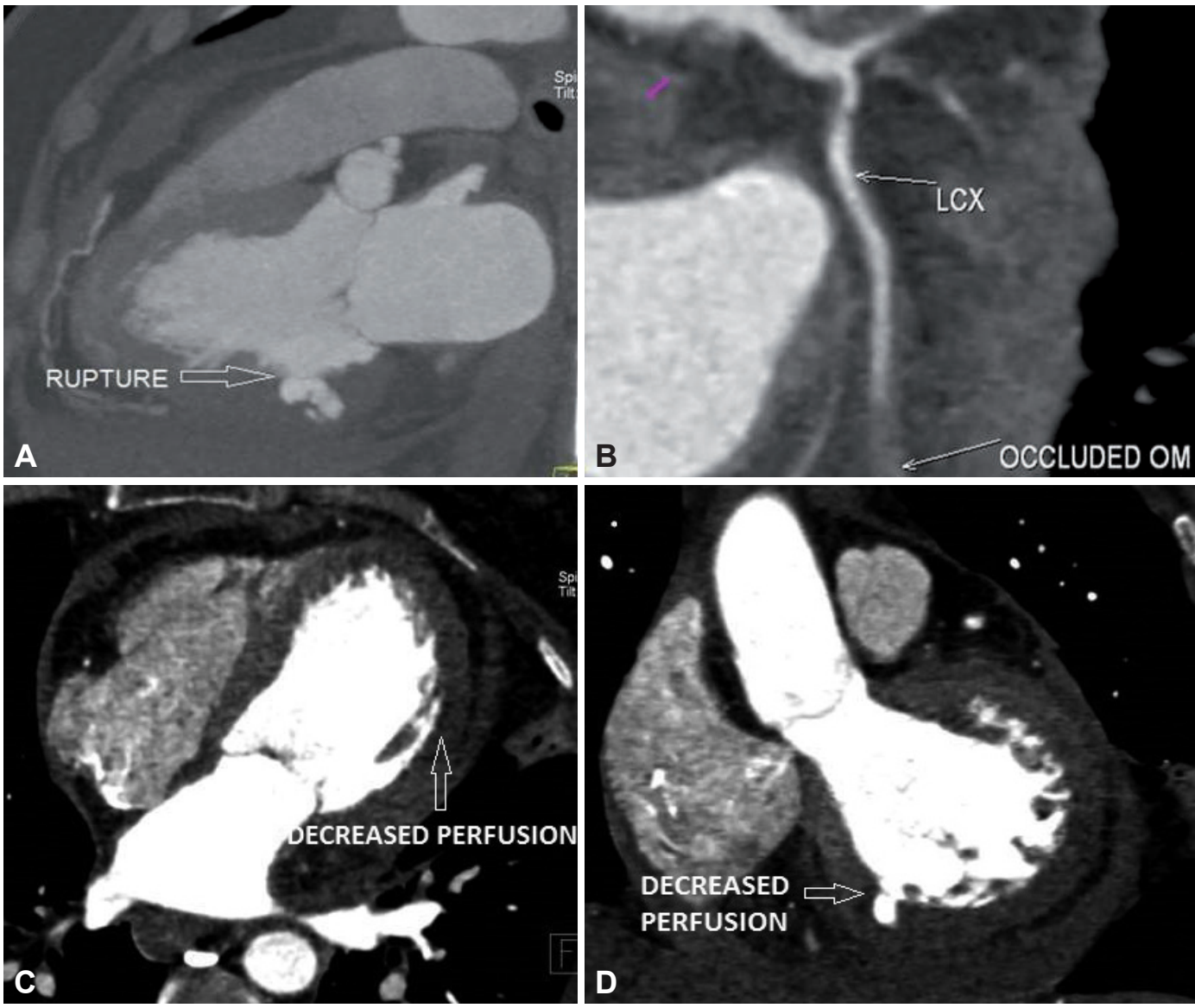

Fig. 1. A 48-year-old female with post-infarct myocardial rupture. (A and B) Maximum intensity projection and curved MPR CT images showing the site of myocardial rupture and occluded OM branch of the LCX. (C and D) Rest perfusion MPR images in the vascular territory of the myocardial rupture. MPR: multiplanar, OM: obtuse marginal, LCX: left circumflex artery.

beats per minute, which was corroborated by the coronary angiogram conducted just prior to confirming the patient for surgery.

The patient underwent emergent surgical repair of cardiac rupture. The pericardial cavity was filled with $500 \mathrm{~mL}$ of altered colored blood. An infarcted and friable myocardium was present in the posterobasal wall, with a rent of $0.5 \mathrm{~cm}$ near the atrioventricular groove. The tear was compatible with the contrast pooling seen on MDCT imaging. The repair was done with a bovine pericardial patch.

Surgery was successful with no peri-procedural or post-operative complications. The patient was subsequently weaned from ventilator support, inotropes, and an intra-aortic balloon pump. She made an uneventful recovery and was discharged in a satisfactory condition.

\section{DISCUSSION}

According to the SHOCK Trial Registry, in-hospital mortality rates of myocardial rupture have reached $60 \%$ [1]. Although the incidence of free wall rupture following myocardial infarc- tion has been reported to be $2.7 \%$, the true rate is difficult to assess due to unconfirmed causes of death, especially pre-hospital death, and diminished autopsy rates [1]. This complication occurs more frequently in patients of the female sex, advanced age, first-time myocardial infarction, elevated blood pressure, and transmural infarction [1]. Ischemic myocardial rupture following acute MI can involve the LV and RV free walls, ventricular septum, and LV papillary muscle, in decreasing order of frequency. The complication rarely involves the left or right atrial walls. The left anterior descending (LAD) artery is the most common culprit artery to this fatal event. In contrast, the right coronary artery (RCA) is relatively uncommon, with a reported incidence of $0-23 \%[1,2]$.

In some patients that survive LV free-wall rupture following acute MI, the rupture can be sealed by the epicardium (visceral pericardium) or by a hematoma on the epicardial surface of the heart. This phenomenon has been referred to as LV diverticulum (or contained myocardial rupture) and represents a subacute pathologic condition between free rupture into the pericardial cavity and formation of a pseudoaneurysm [3].

A pseudoaneurysm is formed if the area of rupture is con- 
tained locally by the adjacent parietal pericardium and represents the chronic stage of LV free wall rupture. The most common etiology of LV pseudoaneurysm has been shown to be myocardial infarction, with inferior infarcts being approximately twice as common as anterior infarcts [4].

Myocardial free wall rupture is associated more frequently with thrombolysis, especially when thrombolysis is delayed by six hours [5]. In the case above, delayed thrombolysis was administered, which could have been a causative factor in the myocardial free wall rupture. Because the rupture was contained by the epicardium, the patient did not present with a cardiac tamponade.

In the diagnosis of cardiac rupture, it is generally difficult to show defects in the ventricular walls. Pericardial effusion by CT or echocardiography is only an indirect indicator of an underlying rupture. In most cases of post-infarct cardiac free wall rupture, the hemodynamic status is unstable. Accordingly, echocardiography is utilized for detecting pericardial effusion because of its rapidity and ease. Hence, there is reluctance on the part of clinicians to utilize cardiac CT for diagnosis.

MDCT provides valuable information in a patient suspected of post-infarct cardiac rupture. The process is rapid, noninvasive, yields information on coronary anatomy, confirms pericardial effusion, and delineates the track of the rupture. Imaging via MDCT also provides information on the rest perfusion status of the infarct-related vessel, the thinness or thickness of the infarcted muscle, and the dynamic visualization of the contrast exiting the LV cavity and entering the pericardial cavity. Existing literature includes few case reports on coincidentally detecting cardiac rupture following myocardial infarction by means of CT when the scan was undertaken to detect other pathology such as aortic dissection. Redfern and Smart [6] reported a case of post-infarct cardiac rupture with a tear at the LV apex detected by CT. The authors found contrast medium in the pericardial cavity, indicating the spilling of blood into the pericardial cavity [6]. Onoda et al. [7] reported a case of cardiac rupture diagnosed on MDCT with small contrast pooling in the posterolateral LV wall within a hypoperfused area. This finding is equivalent to free wall rupture within an infarcted LV wall.

In conclusion, cardiac CT is a noninvasive, accurate, and cost effective tool for the early diagnosis of cardiac rupture with subsequent appropriate surgical treatment, which in turn can improve prognosis in this high-risk subset of patients.

\section{Conflicts of Interest}

The authors declare that they have no conflict of interest.

\section{REFERENCES}

1. Slater J, Brown RJ, Antonelli TA, Menon V, Boland J, Col J, et al. Cardiogenic shock due to cardiac free-wall rupture or tamponade after acute myocardial infarction: a report from the SHOCK Trial Registry. J Am Coll Cardiol 2000;36(3 Suppl A):1117-1122.

2. Nakamura F, Minamino T, Higashino Y, Ito H, Fujii K, Fujita T, et al. Cardiac free wall rupture in acute myocardial infarction: ameliorative effect of coronary reperfusion. Clin Cardiol 1992;15:244-250.

3. Helmy TA, Nicholson WJ, Lick S, Uretsky BF. Contained myocardial rupture: a variant linking complete and incomplete rupture. Heart 2005;91: e13.

4. Frances C, Romero A, Grady D. Left ventricular pseudoaneurysm. J Am Coll Cardiol 1998;32:557-561.

5. Becker RC, Charlesworth A, Wilcox RG, Hampton J, Skene A, Gore JM, et al. Cardiac rupture associated with thrombolytic therapy: impact of time to treatment in the Late Assessment of Thrombolytic Efficacy (LATE) study. J Am Coll Cardiol 1995;25:1063-1068.

6. Redfern A, Smart J. Cardiac rupture. N Engl J Med 2003;348:609.

7. Onoda N, Nonami A, Yabe T, Doi YL, Fujita Y, Yamamoto S, et al. Postinfarct cardiac free wall rupture detected by multidetector computed tomography. J Cardiol Cases 2012;5:e147-e149. 